\title{
EBV-Specific Cytotoxic T-Lymphocyte
}

National Cancer Institute

\section{Source}

National Cancer Institute. EBV-Specific Cytotoxic T-Lymphocyte. NCI Thesaurus. Code C12920.

A white blood cell that is derived from a lymphocyte stem cell matured in the thymus and characterized by a CD8 marker on the surface and an antigen-specific Epstein Barr virus T cell receptor. 